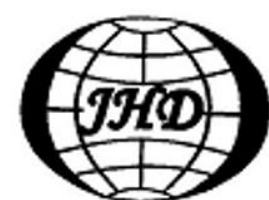

www.sciencedirect.com/ science/journal/10016058

\title{
PREDICTION OF VORTEX-INDUCED VIBRATION OF LONG FLEXIBLE CYLINDERS MODELED BY A COUPLED NONLINEAR OSCILLATOR: INTEGRAL TRANSFORM SOLUTION*
}

\author{
GU Ji-jun, AN Chen, LEVI Carlos \\ Ocean Engineering Program, COPPE, Universidade Federal do Rio de Janeiro, CP 68508, Rio de Janeiro \\ 21941-972, Brazil, E-mail: gu@lts.coppe.ufrj.br \\ SU Jian \\ Nuclear Engineering Program, COPPE, Universidade Federal do Rio de Janeiro, CP 68509, Rio de Janeiro \\ 21941-972, Brazil
}

(Received January 28, 2012, Revised April 10, 2012)

\begin{abstract}
The Generalized Integral Transform Technique (GITT) was applied to predict dynamic response of Vortex-Induced Vibration (VIV) of a long flexible cylinder. A nonlinear wake oscillator model was used to represent the cross-flow force acting on the cylinder, leading to a coupled system of second-order Partial Differential Equations (PDEs) in temporal variable. The GITT approach was used to transform the system of PDEs to a system of Ordinary Differential Equations (ODEs), which was numerically solved by using the Adams-Moulton and Gear method (DIVPAG) developed by the International Mathematics and Statistics Library (IMSL). Numerical results were presented for comparison to those given by the finite difference method and experimental results, allowing a critical evaluation of the technique performance. The influence of variation of mean axial tension induced by elongation of flexible cylinder was evaluated, which was shown to be not negligible in numerical simulation of VIV of a long flexible cylinder.
\end{abstract}

Key words: Vortex-Induced Vibration (VIV), nonlinear wake oscillator, flexible cylinder, integral transform

\section{Introduction}

Vortex-Induced Vibration (VIV) is a major concern in the design of deep-water risers, such as drilling risers, top tensioned risers, and steel catenary risers, since it can result in large amplitude responses in both In-Line (IL) and Cross-Flow (CF) directions, and further lead to accumulation of fatigue damage within a relatively short time period ${ }^{[1,2]}$.

Many research work has been carried out to understand the characteristics of multi-mode VIV, including experimental study ${ }^{[3-5]}$, computational fluid dynamic $\operatorname{codes}^{[6,7]}$, semi-empirical models ${ }^{[8,9]}$ and wake oscillator models ${ }^{[10-12]}$. Due to its simplicity, the wake oscillator model has been employed to perform comprehensive parametrical studies for the VIV of

\footnotetext{
* Biography: GU Ji-jun (1981-), Male, Ph. D.

Corresponding author: SU Jian,

E-mail:sujian@nuclear.ufrj.br
}

flexible cylinders. Instead of direct application of measured fluid forces to structural motion equation, wake oscillator models couple structural motion equation with a nonlinear oscillator equation that describes fluid force. The oscillator models generally have several good characteristics, such as the oscillator is selfexciting and self-limiting, the natural frequency of the oscillator is proportional to the flow velocity and thereby the Strouhal relationship is satisfied, the cylinder motion interacts with the oscillator. Facchinetti et al. ${ }^{[13]}$ systematically evaluated three different coupling terms, that is, acceleration, velocity, and displacement couplings, and concluded that the acceleration coupling can succeed in modeling the features of 2-D VIV. Lin et al. ${ }^{[14]}$ improved the wake oscillator by using a nonlinear fluid damping model, which can predict response amplitude in VIV more accurately than the linear fluid damping model. Xu et al. ${ }^{[15]}$ presented a simple empirical model and studied dynamics of high aspect-ratio $(L / D)$ riser under VIV. Later, $\mathrm{Xu}$ et al. ${ }^{[11]}$ estimated empirical parameters in the 
wake oscillator model for the VIV of an elastically supported cylinder. Violette et al. ${ }^{[16]}$ performed the VIV prediction of a straight slender cylinder oscillating in cross-flow direction under uniform flow, nonuniform flow, and linearly sheared flow, and achieved good agreement with direct numerical simulation and experimental results. Besides cross-flow response, wake oscillator models have been extended successfully to predict in-line dynamic response in recent literature ${ }^{[10,12]}$.

Many different methods have been applied for numerical solution of coupled nonlinear oscillator models, such as the Finite Difference Method $(\mathrm{FDM})^{[10,12,15-17]}$ and Finite Element Method $(\mathrm{FEM})^{[18,19]}$. However, there are no previous study endeavored to perform the vibration analysis of such coupled nonlinear oscillator model based on the Generalized Integral Transform Technique (GITT) approach. Being a hybrid numerical-analytical approach, the most interesting feature of GITT is automatic and straightforward global error control procedure, which makes it particularly suitable for benchmarking purposes. Although GITT has been largely used in natural convection problem ${ }^{[20,21]}$ and 3-D Navier-Stokes equations $^{[22,23]}$, its application in solid and structure mechanics is only at the beginning. Ma et al. ${ }^{[24]}$ applied GITT to solve a transverse vibration problem of an axial moving string and the convergence behavior of integral transform solution was examined. Recently, $\mathrm{An}$ and $\mathrm{Su}^{[25]}$ employed GITT to obtain a hybrid analytical-numerical solution for dynamic response of clamped axially moving beams.

The present contribution aims at advancing this computational tool towards an accurate solution of dynamic response analysis of flexible structures exerted by external forces. The present paper is organized as follows. In the next section, the coupled structure and nonlinear oscillator model is formulated. In the following section, the exact analytical solution is obtained by carrying out integral transform. Numerical results with automatic global accuracy control are then presented, including resonant frequencies, mode numbers and maxima amplitudes, which are illustrated and compared with those given by the $\mathrm{FDM}^{[10]}$ and experiments ${ }^{[1]}$ to verify practicability of the present approach. Finally, the influence of mean axial tension on dynamic response of flexible cylinder is investigated.

\section{Model description}

\subsection{Nonlinear wake oscillator model}

The marine riser can be modeled as a beam with low flexural stiffness. The deflection of a beam is described by means of the Euler-Bernoulli beam equation. As is shown in Fig.1, a Cartesian coordinate system is used, with its origin at one end of the cylinder model , in which the $x$-axis is parallel to flow velocity, the $z$ axis coincides with spanwise axis of the cylinder model in its undeflected configuration, and the $y$ axis is perpendicular to both. The equation for the transverse displacement $Y$ of the cylinder model is given by

$m \frac{\partial^{2} Y}{\partial T^{2}}+\left(r_{s}+r_{f}\right) \frac{\partial Y}{\partial T}-T_{a} \frac{\partial^{2} Y}{\partial Z^{2}}+E I \frac{\partial^{4} Y}{\partial Z^{4}}=\frac{\rho U^{2} D C_{L}}{2}$

where $E I$ denotes the flexural stiffness, $T_{a}$ the applied axial tension, $\rho$ the fluid density, $U$ the fluid velocity, $D$ the diameter of the cylinder model, $C_{L}$ the vortex lift coefficient, $r_{s}$ the structural damping, $r_{f}$ the fluid added damping, $T$ the time, and $Z$ the coordinate in the spanwise direction. The mass $m$ is composed of mass of cylinder model $m_{s}$, internal fluid mass $m_{f}$ and external fluid-added mass $m_{a}$ per unit length, which can be determined by the following relations

$m=m_{s}+m_{f}+m_{a}, \quad m_{f}=\frac{C_{M} \rho D^{2} \pi}{4}, r_{f}=\gamma \Omega \rho D^{2}$

where $C_{M}$ denotes the added mass coefficient, $\Omega$ the reference frequency. In the case of transverse vibration, $\Omega$ is defined by vortex-shedding angular frequency, $\Omega=\Omega_{f}=2 \pi S t U / D$, and $S t$ is the Strouhal number. The fluid-added damping coefficient is $\gamma=C_{D} / 4 \pi S t$, directly related to the mean sectional drag coefficient $C_{D}$ of the structure.

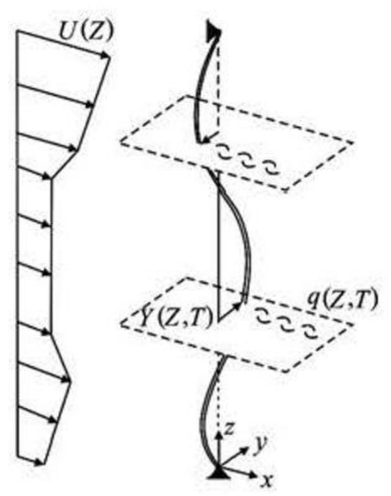

Fig.1 Schematic diagram of the structure-wake oscillators coupling model of VIV

A forced Van Der Pol nonlinear oscillator equa- 
tion is adopted to describe fluid dynamics of fluctuating wake around the cylinders, and an acceleration coupling is selected based on previous work by Faccinetti et al. ${ }^{[13]}$, given as

$$
\frac{\partial^{2} q}{\partial T^{2}}+\varepsilon \Omega_{f}\left(q^{2}-1\right) \frac{\partial q}{\partial T}+\Omega_{f}^{2} q=A \frac{\partial^{2} y}{\partial t^{2}}
$$

where $q$ is reduced fluctuating lift coefficient $q(Z, T)=2 C_{L}(Z, T) / C_{L o}$ and the coefficient $C_{L o}$ denotes amplitude of fluctuating lift for a fixed rigid cylinder subjected to vortex shedding. The values of the Van Der Pol parameter $\varepsilon$ and scaling parameter $A$ can be derived from experimental results from Faccinetti et al. ${ }^{[13]}$. Under the acceleration coupling model, the value of $\varepsilon$ is set as 0.3 according to a best-fitting on the lock-in bands for synchronization of vortex shedding with transverse cylinder vibration. The value of the combined parameter $A / \varepsilon=40$ is proposed from a least-square interpolation between lift magnification and the imposed structure motion amplitude, thus setting $A=12$. By introducing dimensionless mass ratio $\mu=m / \rho D^{2}$, dimensionless time $t=T \Omega_{f}$, transverse displacement $y=Y / D$ and span position $z=Z / L$, the coupled fluid-structure dynamical system Eqs.(1) and (3) turn to be

$$
\begin{aligned}
& \frac{\partial^{2} y}{\partial t^{2}}+\delta \frac{\partial y}{\partial t}-c^{2} \frac{\partial^{2} y}{\partial z^{2}}+b^{2} \frac{\partial^{4} y}{\partial z^{4}}=M q, \\
& \frac{\partial^{2} q}{\partial t^{2}}+\varepsilon\left(q^{2}-1\right) \frac{\partial q}{\partial t}+q=A \frac{\partial^{2} y}{\partial t^{2}}
\end{aligned}
$$

The dimensionless damping $\delta$, tension $c$, bending stiffness $b$ and mass number $M$ are given by

$$
\begin{aligned}
& \delta=\frac{r_{s}}{m \Omega_{f}}+\frac{\gamma}{\mu}, c^{2}=\frac{T_{a}}{m \Omega_{f}^{2} L^{2}}, \quad b^{2}=\frac{E I}{m \Omega_{f}^{2} L^{4}}, \\
& M=\frac{C_{L o}}{2} \frac{1}{8 \pi^{2} S t^{2} \mu}
\end{aligned}
$$

The cylinder model is pin-ended, and hence deflections and curvatures are equal to zero at each end, giving the following boundary conditions:

$$
\begin{aligned}
& y(0, t)=0, \frac{\partial^{2} y(0, t)}{\partial z^{2}}=0, \forall t \\
& y(1, t)=0, \frac{\partial^{2} y(1, t)}{\partial z^{2}}=0, \forall t
\end{aligned}
$$

\subsection{The variation of top tension}

For a pin-ended cylinder towed in a water tank, the applied axial tension $T_{a}$ is in fluctuation due to in-line and cross-flow vibrations. Using Hooke's law, a mean tension $T_{\text {mean }}$ can be defined as

$T_{\text {mean }}=T_{i n i}+E A_{c} \frac{\Delta L}{L}$

where $T_{i n i}$ is initial tension force, $\Delta L=S-L, L$ and $S$ denote initial length and instantaneous length of the cylinder model, respectively, and $A_{c}$ the wall cross section area. For long flexible cylinder, the deflection induced by drag force is much greater than lift force, therefore the elongation is mainly induced by drag force, and the approximate relation reads

$S=\int_{0}^{L} \sqrt{1+Y^{\prime 2}} \mathrm{~d} Z$

For small deflections, $Y^{\prime}$ is sufficiently small to allow Eq.(8) to be approximated as

$S=\int_{0}^{L}\left(1+\frac{1}{2} Y^{\prime 2}\right) \mathrm{d} Z$

Simplify the equation yields

$\Delta L=\frac{1}{2} \int_{0}^{L} Y^{\prime 2} \mathrm{~d} Z$

For a pin-ended beam, an analytical solution can be found if deflection shape is represented by a sine series

$$
Y=\frac{P}{\frac{E I \pi^{4}}{L^{4}}+T_{\text {mean }} \frac{\pi^{2}}{L^{2}}} \sin \frac{\pi Z}{L}
$$

where $P$ is external force exerted perpendicularly on the model. Here only deflection induced by drag force is considered, and therefore, $P$ can be approximately expressed as

$$
P=\frac{1}{2} \rho U^{2} D C_{D}
$$

The mean tension, related to flow velocity $U$, is finally obtained by combining Eqs.(7), (10), (11) and (12)

$$
T_{\text {mean }}=T_{i n i}+\frac{E A_{c}\left(\rho U^{2} D C_{D} L\right)^{2}}{16\left(E I \pi^{2}+T_{\text {mean }} L^{2}\right)}
$$




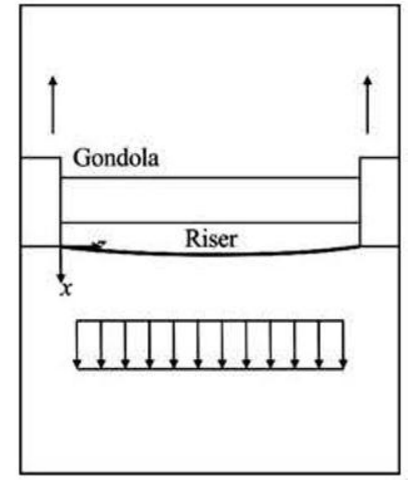

Fig.2 Overall layout of the VIV experiment with the carriage speed of $0.3 \mathrm{~m} / \mathrm{s}-2.4 \mathrm{~m} / \mathrm{s}$ at Marintek ${ }^{[1]}$

\section{Integral transform solution}

Following the ideas in the GITT, the next step is that of selecting eigenvalue problem and proposing eigenfunction expansion. The eigenvalue problem for transverse displacement $y(z, t)$ of the cylinder model is chosen as

$\frac{\mathrm{d}^{4} \phi_{i}(z)}{\mathrm{d} z^{4}}=\alpha_{i}^{4} \phi_{i}(z), \quad 0<z<1$

Together with the following boundary conditions

$\phi_{i}(0)=0, \quad \phi_{i}(1)=0, \frac{\mathrm{d}^{2} \phi_{i}(0)}{\mathrm{d} z^{2}}=0, \frac{\mathrm{d}^{2} \phi_{i}(1)}{\mathrm{d} z^{2}}=0$

where $\phi_{i}(z)$ is the eigenfunction of problem (14) corresponding to the eigenvalue $\alpha_{i}$, satisfying the following orthogonality property

$\int_{0}^{1} \phi_{i}(z) \phi_{j}(z) \mathrm{d} z=\delta_{i j} N_{i}$

where with $\delta_{i j}$ is the Kronecker delta. The norm, or normalization integral, is written as

$N_{i}=\int_{0}^{1} \phi_{i}^{2}(z) \mathrm{d} z$

Problem (14) is readily solved analytically to yield

$\phi_{i}(z)=\sin \left(\alpha_{i} z\right)$

and the eigenvalue becomes

$\alpha_{i}=\mathrm{i} \pi, \quad i=1,2,3, \ldots$ while the norm is evaluated to yield

$N_{i}=\frac{1}{2}, \quad i=1,2,3, \ldots$

The eigenvalue problem (14) allows the definition of the following integral transform pairs:

$\bar{y}_{i}(t)=\int_{0}^{1} \tilde{\phi}_{i}(z) y(z, t) \mathrm{d} z$, transform

$y(z, t)=\sum_{i=1}^{\infty} \tilde{\phi}_{i}(z) \bar{y}_{i}(t)$, inversion

where $\tilde{\phi}_{i}(z)$ is the normalized eigenfunction

$\tilde{\phi}_{i}(z)=\frac{\phi_{i}(z)}{N_{i}^{1 / 2}}=\sqrt{2} \phi_{i}(z)$

The reduced lift coefficient $q(z, t)$ of the cylinder model is chosen as

$\frac{\mathrm{d}^{2} \psi_{k}(z)}{\mathrm{d} z^{2}}=-\beta_{k}^{2} \psi_{k}(z), \quad 0<z<1$

where $\psi_{k}(z)$ is the eigenfunction of problem (23), and $\beta_{k}$ the corresponding eigenvalue. Then the same mathematical manipulation is carried out as for Eqs.(15)-(20), and the eigenvalue problem (23) allows the definition of the following integral transform pairs, where $\tilde{\psi}_{k}(z)$ is the normalized eigenfunction.

$\bar{q}_{k}(t)=\int_{0}^{1} \tilde{\psi}_{k}(z) q(z, t) \mathrm{d} z$, transform

$q(z, t)=\sum_{k=1}^{\infty} \tilde{\psi}_{k}(z) \bar{q}_{k}(t)$, inversion

Now, to perform integral transform process, the dimensionless form of Eq.(4) are multiplied by operator $\int_{0}^{1} \tilde{\phi}_{i}(z) \mathrm{d} z$ and $\int_{0}^{1} \tilde{\psi}_{k}(z) \mathrm{d} z$, respectively, the inverse formula (21) and (24) are applied, yielding, after some mathematical manipulations, the following set of ordinary differential equations:

$\frac{\mathrm{d}^{2} \bar{y}_{i}(t)}{\mathrm{d} t^{2}}+\delta \frac{\mathrm{d} \bar{y}_{i}(t)}{\mathrm{d} t}-c^{2} \sum_{j=1}^{\infty} \tilde{P}_{i j} \bar{y}_{j}(t)+b^{2} \alpha_{i}^{4} \bar{y}_{i}(t)=$

$M \sum_{k=1}^{\infty} \tilde{Q}_{i k} \bar{q}_{k}(t), \quad i=1,2,3, \ldots$ 
Table 1 The main parameters of VIV experiments performed by Trim et al. ${ }^{[1]}$

\begin{tabular}{|c|c|c|c|c|c|c|c|c|c|}
\hline $\begin{array}{l}\text { Total } \\
\text { length, } \\
L \quad(\mathrm{~m})\end{array}$ & $\begin{array}{c}\text { Outer } \\
\text { diameter, } \\
D(\mathrm{~m})\end{array}$ & $\begin{array}{c}\text { Inner } \\
\text { diameter, } \\
d \quad(\mathrm{~m})\end{array}$ & $\begin{array}{l}\text { Bending } \\
\text { stiffness, } \\
\text { EI }\left(\mathrm{Nm}^{2}\right)\end{array}$ & $\begin{array}{c}\text { Mass } \\
\text { ratio, } \\
\mu\end{array}$ & $\begin{array}{c}\text { Aspect } \\
\text { ratio, } \\
\Lambda\end{array}$ & $\begin{array}{c}\text { Top } \\
\text { tension, } \\
T_{a}(\mathrm{~N})\end{array}$ & $\begin{array}{c}\text { Flow } \\
\text { speed, } \\
U \\
(\mathrm{~m} / \mathrm{s})\end{array}$ & $\begin{array}{c}\text { Reynolds } \\
\text { number, } \\
\quad R e\end{array}$ & $\begin{array}{l}\text { Damping } \\
\text { ratio, } \xi\end{array}$ \\
\hline 38 & 0.027 & 0.021 & 37.2 & 1.62 & 1407 & $4000-6000$ & $0.3-2.4$ & $8100-64800$ & 0.01 \\
\hline
\end{tabular}

$$
\begin{gathered}
\frac{\mathrm{d}^{2} \bar{q}_{k}(t)}{\mathrm{d} t^{2}}+\varepsilon \sum_{l=1}^{\infty} \sum_{r=1}^{\infty} \sum_{s=1}^{\infty} \tilde{R}_{k l r s} \bar{q}_{l}(t) \bar{q}_{r}(t) \frac{\mathrm{d} \bar{q}_{s}(t)}{\mathrm{d} t}- \\
\varepsilon \frac{d \bar{q}_{k}(t)}{d t}+\bar{q}_{k}(t)=A \sum_{i=1}^{\infty} \tilde{S}_{k i} \frac{\mathrm{d}^{2} \bar{y}_{i}(t)}{\mathrm{d} t^{2}} \\
k=1,2,3, \ldots
\end{gathered}
$$

where the coefficients are analytically determined from the following integrals:

$$
\begin{aligned}
& \tilde{P}_{i j}=\int_{0}^{1} \tilde{\phi}_{i}(z) \frac{\mathrm{d}^{2} \tilde{\phi}_{j}(z)}{\mathrm{d} z^{2}} \mathrm{~d} z, \\
& \tilde{Q}_{i k}=\int_{0}^{1} \tilde{\phi}_{i}(z) \tilde{\psi}_{k}(z) \mathrm{d} z, \\
& \tilde{R}_{k l r s}=\int_{0}^{1} \tilde{\psi}_{k}(z) \tilde{\psi}_{l}(z) \tilde{\psi}_{r}(z) \tilde{\psi}_{s}(z) \mathrm{d} z, \\
& \tilde{S}_{k i}=\int_{0}^{1} \tilde{\psi}_{k}(z) \tilde{\phi}_{i}(z) \mathrm{d} z
\end{aligned}
$$

In a similar manner, the boundary conditions are also integral transformed in the $z$ direction to yield

$$
\begin{aligned}
& \bar{y}_{i}(0)=0, \quad \bar{y}_{i}(1)=0, \frac{\mathrm{d}^{2} \bar{y}_{i}(0)}{\mathrm{d} t^{2}}=0, \frac{\mathrm{d}^{2} \bar{y}_{i}(1)}{\mathrm{d} t^{2}}=0, \\
& \bar{q}_{k}(0)=0, \quad \bar{q}_{k}(1)=0, \quad \frac{\mathrm{d}^{2} \bar{q}_{k}(0)}{\mathrm{d} t^{2}}=0,
\end{aligned}
$$$$
\frac{\mathrm{d}^{2} \bar{q}_{k}(1)}{\mathrm{d} t^{2}}=0
$$

The equation system (25) is now in the appropriate form for numerical solution through dedicated routines for initial value problems, such as the AdamsMoulton and Gear method (DIVPAG) from International Mathematics and Statistics Library (IMSL), which are well-tested and capable of handling such situations, offering an automatic accuracy control scheme, and for this problem the error $10^{-6}$ is selected. For this computational purpose, the expansions for the transverse displacement $y(z, t)$ and reduced lift coefficient $q(z, t)$ are then truncated to $N$ orders, so as to reach the user requested accuracy target in the final solution. The related coefficients given by Eq.(26) are also handled well through IMSL Library. Once $\bar{y}_{i}(t)$ have been numerically evaluated, the analytical inversion formula (21) recovers the dimensionless function $y(z, t)$.

\section{Results and discussion}

\subsection{Case study}

To validate the GITT solution, numerical simulation of the experiments on a towed riser model in uniform flow conducted by Trim et al. ${ }^{[1]}$ is carried out. The experimental investigation was performed at the Marintek Ocean Basin in Trondheim. The overall layout of the experiments is shown in Fig.2. The riser model was $38 \mathrm{~m}$ in length and $0.027 \mathrm{~m}$ in diameter with an aspect-ratio of 1407 . It was equipped with a dense array of high-quality instrumentation, and the flow profile was uniform stepped from $0.3 \mathrm{~m} / \mathrm{s}$ to $2.4 \mathrm{~m} / \mathrm{s}$ with an increasing step of $0.1 \mathrm{~m} / \mathrm{s}$. The summary of main parameters of the experiments is given in Table 1.

We now present numerical results for transverse displacement $Y(Z, T)$ of the long flexible cylinder by employing the GITT approach. For all initial conditions, a random noise with amplitude of order $O\left(10^{-3}\right)$ is applied to fluid variable $\bar{q}^{[16]}$. Zero $\bar{y}$ and $\bar{y}^{\prime}$ initial conditions are applied to the structure. The added mass coefficient $C_{M}$ is dependent on flow velocity, cylinder vibration amplitude, etc.. Hence, it is difficult to evaluate its value. The constant value of $C_{M}=1.0$ is taken as was done by $\mathrm{Ge}$ et al. ${ }^{[10,12]}$. The coefficient $C_{L}$ is usually taken as 0.3 in the large range of $R e$, and $C_{D}$ is set as 1.2 for a rigid cylinder at the subcritical range, $300<R e<$ $1.5 \times 10^{5[10,12,13]}$. For a cylinder undergoing vibration, the Strouhal number is set as 0.17 . This value is lower than the usually-quoted 0.2 , but has been found to apply for moving cylinders ${ }^{[1]}$.

The solution of system (25) is obtained with maximum truncation order $N=50$ to analyze the convergence behavior. 


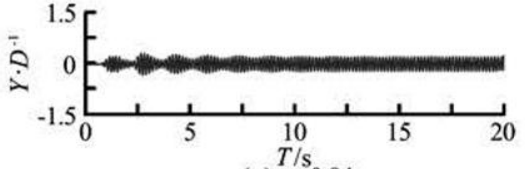

(a) $\stackrel{T / \mathrm{s}}{z=0.84}$

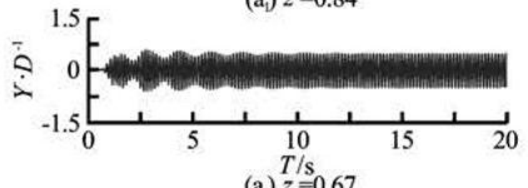

(a) $z=0.67$

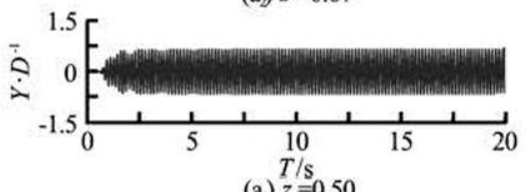

(a) $\stackrel{T / \mathrm{s}}{z=0.50}$

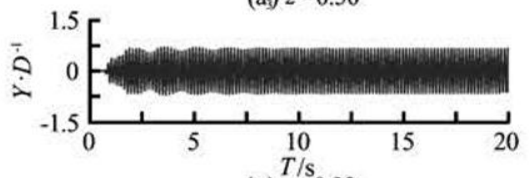

(a) $\stackrel{T / \mathrm{s}}{z=0.33}$

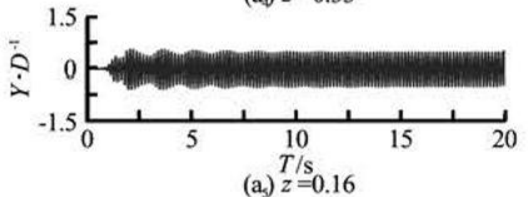

(a)

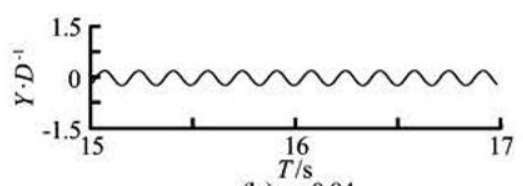

(b) $z=0.84$

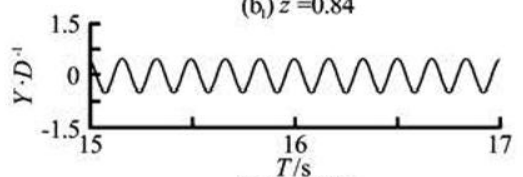

(b) $z=0.67$

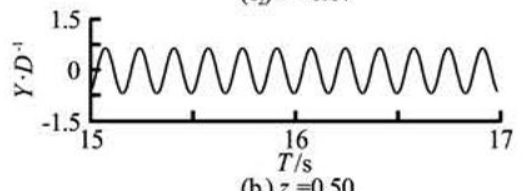

(b) $z=0.50$

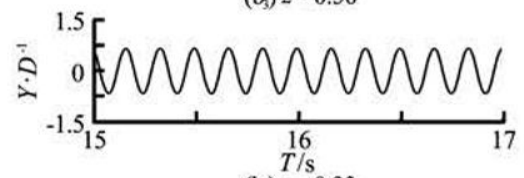

$\left(\mathrm{b}_{4}\right) z=0.33$

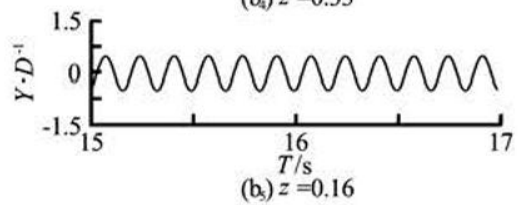

(b)
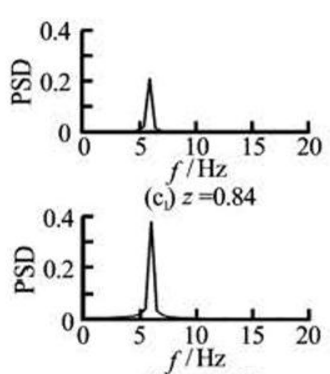

(c) $z=0.67$

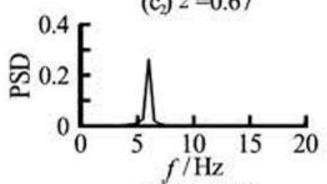

(c) $z=0.50$
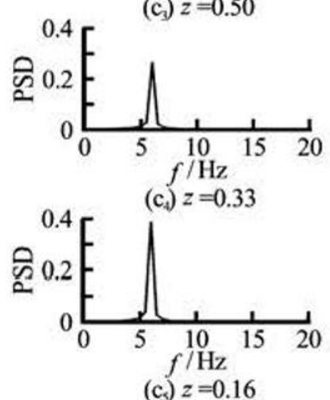

(c)

Fig.3 Time history of GITT solutions with truncation order $N=40$ at $U=1.0 \mathrm{~m} / \mathrm{s}$ (a) Time history of displacement-to-diameter ratio of the whole run at 5 equidistant space interval along the spanwise direction of the cylinder, (b) Time history of displacement-to-diameter ratio in a time interval $T \in[15,17] \mathrm{s}$, (c) Spectral analysis of (b)

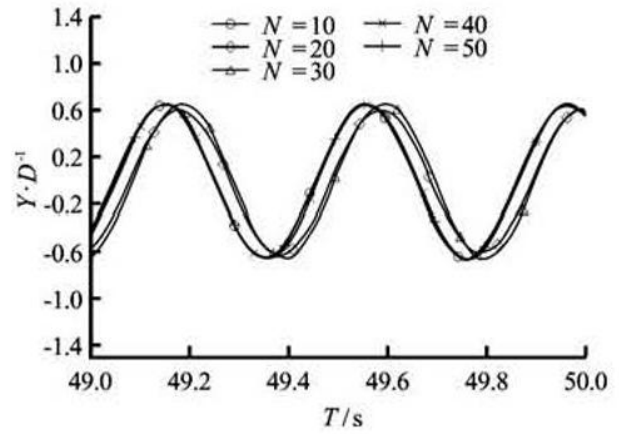

Fig.4 GITT solutions with different truncation orders $N$ for the time history of displacement-to-diameter ratio at $U=0.4 \mathrm{~m} / \mathrm{s}$

The typical time histories and response frequencies are shown in Fig.3, at $U=1.0 \mathrm{~m} / \mathrm{s}$ for the truncation order $N=40$. The first column is the time history of displacement-to-diameter ratio of the whole run at 5 equidistant space interval along the spanwise direction of the cylinder, the second column is time history of displacement-to-diameter ratio in a time interval $T \in[15,17] \mathrm{s}$, and the third column is spectral analysis of second column. It can be observed that the vibration of displacement arrives at a stable state after
$10 \mathrm{~s}$. Only one peak of vibration frequency appears in each result of the spectral analysis, which means that there is a unique mode contributing to the response.

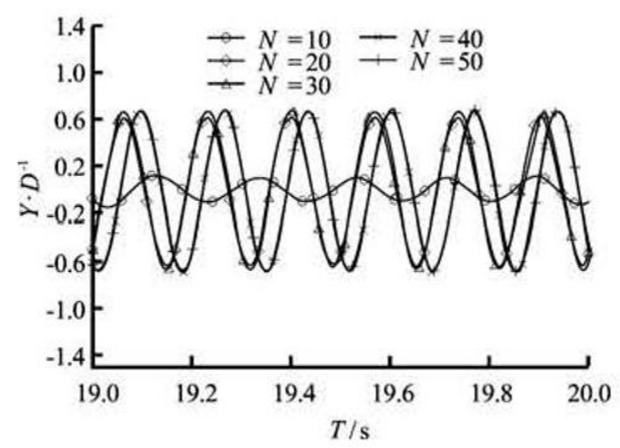

Fig.5 GITT solutions with different truncation orders $N$ for the time history of displacement-to-diameter ratio at $U=1.0 \mathrm{~m} / \mathrm{s}$

The convergence behavior of integral transform solution is examined by increasing truncation orders $N=10,20,30,40$ and 50 . The time traces of $U=$ $0.4 \mathrm{~m} / \mathrm{s}$ at $T \in[49.0,50.0] \mathrm{s}$ and $U=1.0 \mathrm{~m} / \mathrm{s}$ at $T \in[19.0,20.0] \mathrm{s}$ are shown in Figs. 4 and 5. For transverse displacements with $U=0.4 \mathrm{~m} / \mathrm{s}$ and $U=$ $1.0 \mathrm{~m} / \mathrm{s}$, the convergence is quite favorable, demon- 
strated by time traces of $N=40$ and $N=50$ being nearly overlapped. However, it should be pointed out that very low truncation orders may not capture actual displacement, such as the one in Fig.5 with $N=10$.

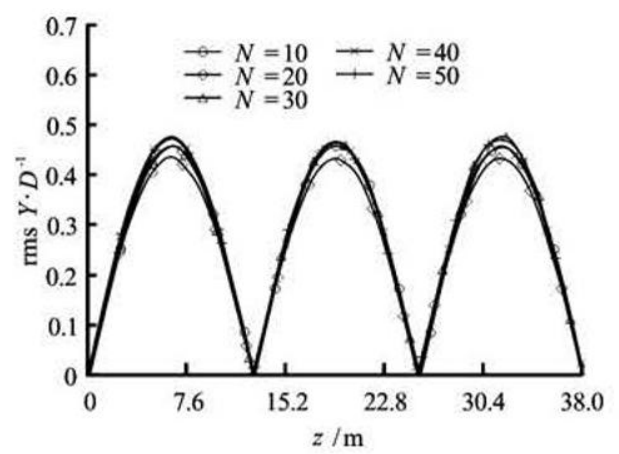

Fig.6 GITT solutions with different truncation orders $N$ for rms of displacement-to-diameter ratio at $U=0.4 \mathrm{~m} / \mathrm{s}$

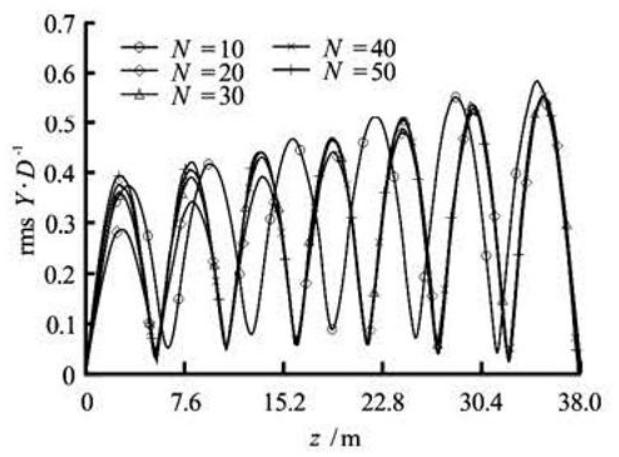

Fig.7 GITT solutions with different truncation orders $N$ for rms of displacement-to-diameter ratio at $U=1.0 \mathrm{~m} / \mathrm{s}$

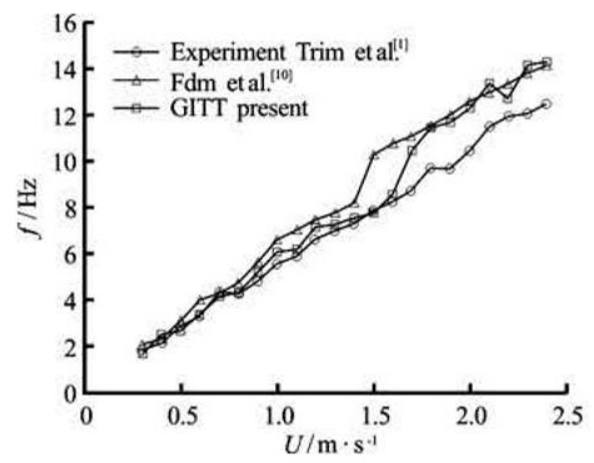

Fig.8 Resonant frequency of the flexible cylinder vibration versus towing speed

The root mean square (rms) of the transverse displacement in a time interval is popularly used in VIV analysis to evaluate vibration amplitude and mode numbers. Figures 6 and 7 depict the rms of transverse displacements at $U=0.4 \mathrm{~m} / \mathrm{s}$ and $U=1.0 \mathrm{~m} / \mathrm{s}$, respectively. Note that with increasing truncation order $N$, the rms values along the cylinder spanwise direction approach a stable state and mode numbers do not change at the higher truncation orders.

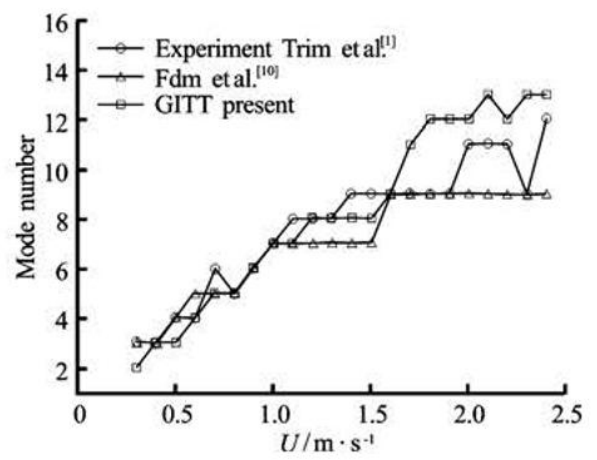

Fig.9 Dominant mode number of the flexible cylinder vibration versus towing speed

The comparison of the resonant frequencies and mode numbers obtained by the proposed approach and those reported by $\mathrm{Ge}$ et al. ${ }^{[10]}$ is performed to verify the accuracy of GITT solution, as shown in Figs. 8 and 9. Most of resonant frequencies from GITT solution are in good agreement with those from the $\mathrm{FDM}^{[10]}$ and experiments ${ }^{[1]}$. The resonant frequencies are higher about $10 \%$ compared with the measuring frequencies at higher flow velocity. As for the prediction of mode numbers, the results from GITT show good agreement with the measuring mode numbers when flow velocity is lower than $1.6 \mathrm{~m} / \mathrm{s}$. Above the value of $1.6 \mathrm{~m} / \mathrm{s}$, the prediction of mode numbers is overestimated by the factor 1-3, which may result from insufficient consideration of structural model, such as the effect of variable added mass coefficient and mean axial tension. The added mass coefficient is dependent on various factors, such as flow velocity and cylinder vibration amplitude. Hence it is difficult to evaluate this value accurately. The influence of mean axial tension will be detailedly discussed in Subsection 3.2.

Figure 10 indicates the instantaneous displacements in one period within time interval $0.0126 \mathrm{~s}$ at $U=0.4 \mathrm{~m} / \mathrm{s}$ (the first row), $0.0072 \mathrm{~s}$ at $U=$ $0.7 \mathrm{~m} / \mathrm{s}$ (the second row) and $0.0034 \mathrm{~s}$ at $U=$ $1.5 \mathrm{~m} / \mathrm{s}$ (the third row), respectively. The stable instantaneous mode shapes show clearly standard standing waves with mode numbers of 3,5 and 8 , respectively. Although multi-modal VIV was observed in some tests such as those by Trim et al. ${ }^{[1]}$ and Chaplin et al. ${ }^{[3]}$, the dynamic response was still dominated by one mode number. The present result depicts that the vibration of cylinder is locked on pure single modal rather than multi-modal one, hence it could not represent full information of multi-modal VIV. Nevertheless, this single modal should be the dominated mode number which has much greater energy than other mode numbers. The maxima amplitudes $\left(Y_{M} / D\right)$ of transverse displacements are shown in Fig.11, as well 

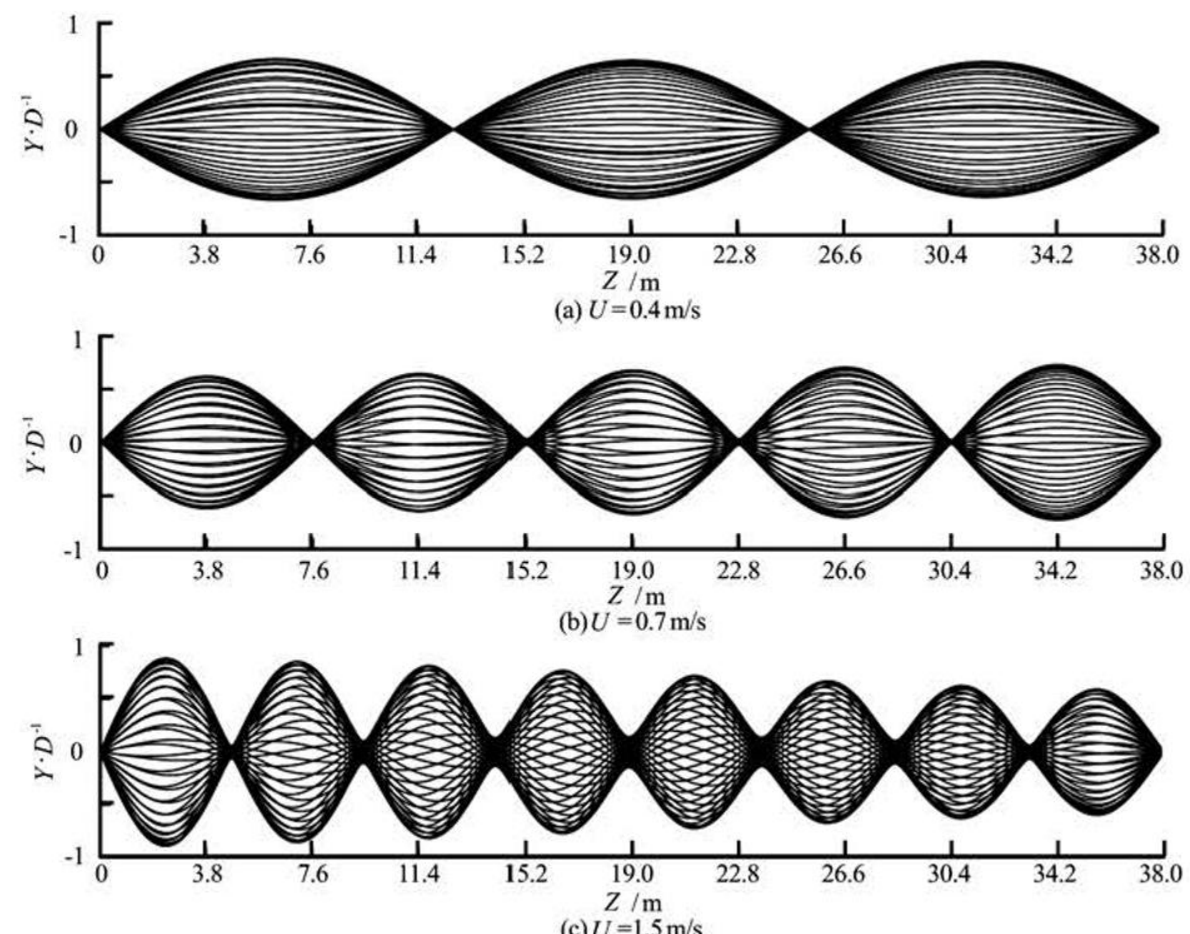

Fig.10 Instantaneous displacements of the flexible riser in one period with time interval $0.0126 \mathrm{~s}$ at $U=0.4 \mathrm{~m} / \mathrm{s}$ (the first row), $0.0072 \mathrm{~s}$ at $U=0.7 \mathrm{~m} / \mathrm{s}$ (the second row) and $0.0034 \mathrm{~s}$ at $U=1.5 \mathrm{~m} / \mathrm{s}$ (the third row)

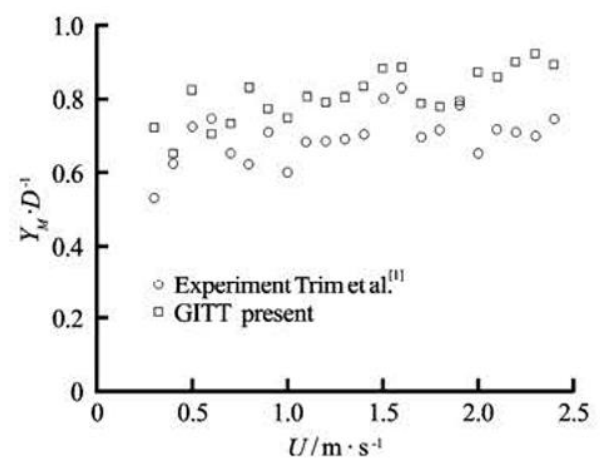

Fig.11 Maximum vibration amplitudes of the flexible riser versus towing speed

as the comparison with measurement data. It can be seen that maximum amplitudes increase slightly as flow velocity increases, which are around $10 \%$ larger than the amplitudes of the corresponding experimental results.

\subsection{Influence of mean axial tension}

The relation between mean axial tension and flow velocity given by Eq.(13) is validated by comparison with the experimental data ${ }^{[26]}$, as shown in Fig.12, where $U_{r}$ is the reduced velocity defined by $U_{r}=(U D) / f_{n}$, where $f_{n}$ is the fundamental natural frequency. The dashed lines are calculated by Eq.(13), and good agreements can be observed. Figure 13 depicts the mean axial tension variation of the case considered in Subsection 3.1. The values of the mean axial tension increase from initial $6000 \mathrm{~N}$ to $10625 \mathrm{~N}$ as flow velocity arrives at its maximum value of $2.4 \mathrm{~m} / \mathrm{s}$.

The effect of the mean axial tension on the dynamic response of flexible cylinder is investigated. Figure 14 shows the plots of model numbers versus flow velocity with different applied axial tensions. The line with triangle symbol represents the FDM results with the constant axial tension of $5000 \mathrm{~N}$ simulated by $\mathrm{Xu}$ et al. ${ }^{[15]}$, the line with the diamond symbol represents the GITT solution considering the constant axial tension of $6000 \mathrm{~N}$, and the line with the square symbol represents the GITT simulations with variable mean axial tensions based on the Eq.(13).

In Fig.14, it can be observed that at the flow velocity $U<1.1 \mathrm{~m} / \mathrm{s}$, GITT solutions have good agreement with experimental results by considering whether or not the variation of mean axial tension. However, when $U>1.1 \mathrm{~m} / \mathrm{s}$, the mode numbers obtained by the GITT with the variation of mean axial tension give more accurate information. The phenomenon can be explained by the fact that the mean axial tension does not change too much at the lowflow-velocity range, as shown in Fig.13, therefore, a constant mean axial tension can be adopted to approximately simulate the real case. When the flow 


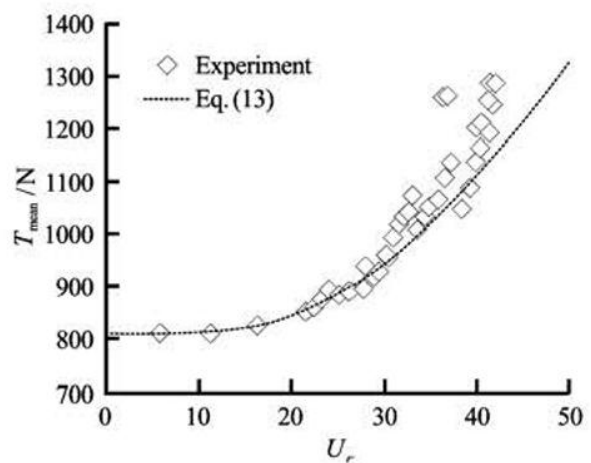

(a) $T_{b e v i}=810 \mathrm{~N}$

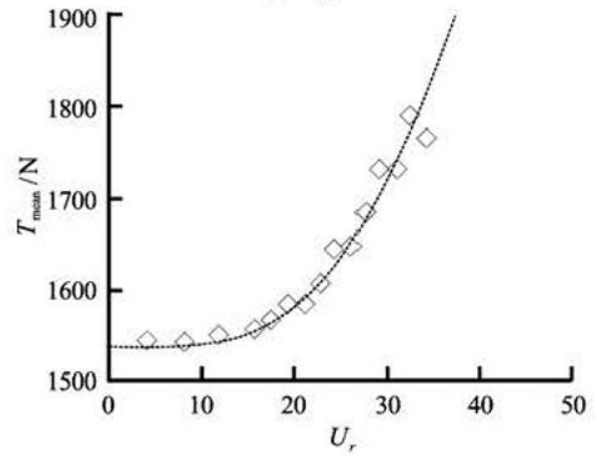

(c) $T_{i s i}=1538 \mathrm{~N}$

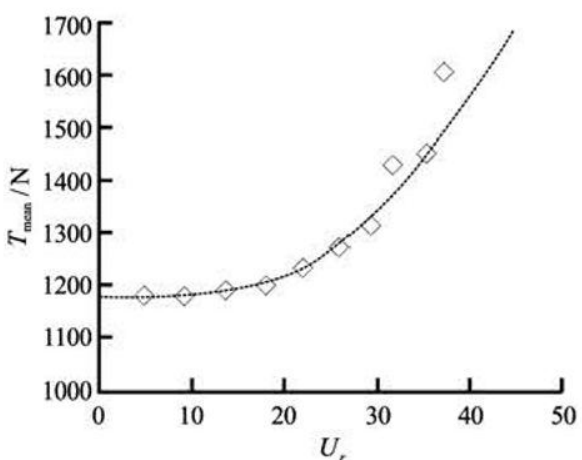

(b) $T_{i n d}=1175 \mathrm{~N}$

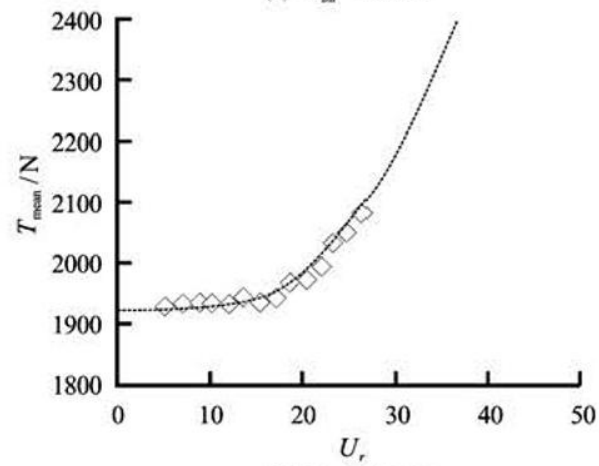

(d) $T_{i n d}=1922 \mathrm{~N}$

Fig.12 Comparison of the mean axial tension obtained by Eq.(13) and from experiments

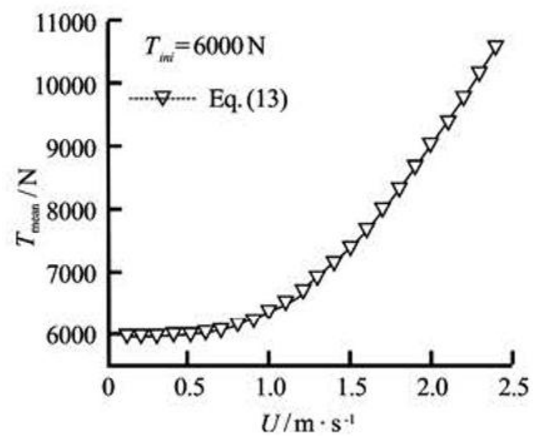

Fig.13 Mean axial tension obtained by Eq.(13) for the experiment carried out by Trim et al. ${ }^{[1]}$

velocity is quite higher, the constant mean axial tension fails to represent the top tension exactly. From the theory of vibration, we know that an increased axial tension can increase natural frequency of cylinder model at a specific mode number. To excite structure to yield the same mode number, the frequency of excitation (i.e., the vortex shedding frequency) needs to increase correspondingly, which means a higher towing speed should be applied. In other words, when towing speed is not changed, the frequency of excitation only can be "controlled" at a lower mode number, which explains why the mode number does not increase linearly with towing speed increasing. Besides, it shows that both the GITT solutions agree better with the experimental results than the one given by $\mathrm{Xu}$ et al. ${ }^{[15]}$ which did not consider the axial tension variation.

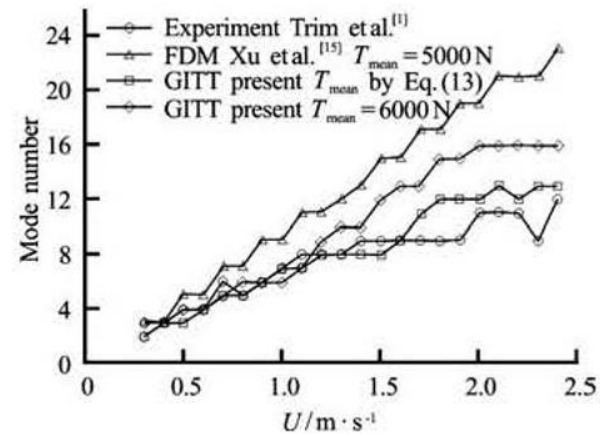

Fig.14 Dominant mode number of the flexible cylinder vibration versus towing speed

However, the prediction of mode numbers is still overestimated by a factor 1-3 through the GITT approach when flow velocity $U>1.6 \mathrm{~m} / \mathrm{s}$ as shown in Fig.9. In addition to the uncertainty of added mass, the underestimate of mean axial tension at high flow velocity may be another reason. Although Fig.12 shows good simulation of top tension by Eq.(13), there still exists a microscopic underestimate of mean axial tension when $T_{i n i}=810 \mathrm{~N}$ and $T_{i n i}=1175 \mathrm{~N}$. As for the present case, the mean axial tension may be grea- 
ter than the one obtained from Eq.(13) as shown in Fig.13, especially when flow velocity $U>1.6 \mathrm{~m} / \mathrm{s}$, which can induce the overestimate of prediction of mode numbers by a factor 1-3. Hence, the prediction of mode numbers is quite sensitive to the mean axial tension.

\section{Conclusions}

In this paper, a GITT has been proposed for the solution of VIV prediction of a long flexible cylinder using a nonlinear oscillator. The analysis of convergence behavior and comparisons between experimental results and numerical simulation has shown that this hybrid numerical-analytical approach is adequate to deal with such nonlinear coupling system. The following conclusions can be drawn:

(1)The generalized integral transform technique exhibits good convenience behavior as the truncation order approaches 50.

(2)The solution from GITT of wake oscillator model is capable of evaluating multi-mode lock-in response, dominant resonant frequencies and mode numbers, which are in good agreement with the FDM and experimental results.

(3)The program evaluates instantaneous displacement with standard standing waves, and the maximum amplitudes are around $10 \%$ larger than the amplitudes of the corresponding experimental results.

(4)The increase of mean axial tension can decrease the mode number of response, and the relation with initial axial tension is yielded as Eq.(13), therefore, its variation induced by elongation of flexible cylinder cannot be neglected in such numerical simulation.

This investigation verifies that the proposed GITT approach can be performed in dynamic response of string-like structures coupled with environmental loads, such as current, wind or waves in offshore. Due to the simplicity of wake oscillator model, all results presented in this paper require a short period of computational time, which is more practicable than CFD methods.

\section{Acknowledgments}

The authors acknowledge gratefully financial support provided by CNPq, CAPES and FAPERJ of Brazil for their research work. Gu Ji-jun and An Chen also would like to acknowledge financial support provided by China Scholarship Council.

\section{References}

[1] TRIM A. D., BRAATEN H. and LIE H. et al. A. Experimental investigation of vortex-induced vibration of long marine risers[J]. Journal of Fluids and Structures, 2005, 21(3): 335-361.

[2] GAO Yun, ZONG Zhi and SUN Lei. Numerical prediction of fatigue damage in steel catenary riser due to vortex-induced vibration[J]. Journal of Hydrodynamics, 2011, 23(2): 154-163.

[3] CHAPLIN J. R., BEARMAN P. W. and HUERAHUARTE F. J. et al. Laboratory measurements of vortex-induced vibrations of a vertical tension riser in a stepped current[J]. Journal of Fluids and Structures, 2005, 21(1): 3-24.

[4] VANDIVER J. K., SWITHENBANK S. B. and JAISWAL V. et al. Fatigue damage from high mode number vortex-induced vibration[C]. Proceedings of the 25th International Conference on Offshore Mechanics and Arctic Engineering. Humburg, Gemany, 2006, 4: 803-811.

[5] HUANG Shan, SWORN Andy. Some observations of two interfering VIV circular cylinders of unequal diameters in tandem[J]. Journal of Hydrodynamics, 2011, 23(5): 535-543.

[6] YAMAMOTO C. T., MENEGHINI J. R. and SALTARA F. et al. Numerical simulations of vortex-induced vibration on flexible cylinders[J]. Journal of Fluids and Structures, 2004, 19(4): 467-489.

[7] XIE Fang-fang, DENG Jian and ZHENG Yao. Multimode of vortex-induced vibration of a flexible circular cylinder[J]. Journal of Hydrodynamics, 2011, 23(4): 483-490.

[8] NARAKORN S. MARIAN W. and PATRICK O. Reduced-order modeling of vortex-induced vibration of catenary riser[J]. Ocean Engineering, 2009, 36(17-18): 1404-1414.

[9] NARAKORN S. Multi-mode interactions in vortex-induced vibrations of flexible curved/straight structures with geometric nonlinearities[J]. Journal of Fluids and Structures, 2010, 26(7-8): 1098-1122.

[10] GE Fei, LONG Xu and WANG Lei et al. Flow-induced vibrations of long circular cylinders modeled by coupled nonlinear oscillators[J]. Science in China, Series G-Physics Mechanics and Astronomy, 2009, 52(7): 1086-1093.

[11] XU M.-R., XU S.-P. and GUO H.-Y. Determination of natural frequencies of fluid-conveying pipes using homotopy perturbation method[J]. Computers and Mathematics with Applications, 2010, 60(3): 520527.

[12] GE Fei, LU Wei and WANG Lei et al. Shear flow induced vibrations of long slender cylinders with a wake oscillator model[J]. Acta Mechanica Sinica, 2011, 27(3): 330-338.

[13] FACCHINETTI M. L., De LANGRE E. and BIOLLEY F. Coupling of structure and wake oscillators in vortexinduced vibrations[J]. Journal of Fluids and Structures, 2004, 19(2): 123-140.

[14] LIN Li-ming, LING Guo-can and WU Ying-xiang et al. Nonlinear fluid damping in structure-wake oscillators in modeling vortex-induced vibrations[J]. Journal of Hydrodynamics, 2009, 21(1): 1-11.

[15] XU Wan-Hai, ZENG Xiao-Hui and WU Ying-xiang. High aspect ratio (L/D) riser VIV prediction using wake oscillator model[J]. Ocean Engineering, 2008, 35(1718): 1769-1774.

[16] VIOLETTE R., De LANGRE E. and SZYDLOWSKI J. Computation of vortex-induced vibrations of long structures using a wake oscillator model: Comparison with 
DNS and experiments[J]. Computers and Structures, 2007, 85(11-14): 1134-1141.

[17] FACCHINETTI M. L., De Langre E. and BIOLLEY F. Vortex-induced travelling waves along a cable[J]. European Journal of Mechanics B-Fluids, 2004, 23(1): 199-208.

[18] LOU Min, DING Jian and GUO Hai-yan et al. Effect of internal flow on vortex-induced vibration of submarine free spanning pipelines[J]. China Ocean Engineering, 2005, 19(1): 147-154.

[19] GUO Hai-yan, LI Xiao-min and LIU Xiao-chun. Numerical prediction of vortex-induced vibrations on top tensioned riser in consideration of internal flow[J]. China Ocean Engineering, 2008, 22(4): 675-682.

[20] LEAL M. A., MACHADO H. A. and COTTA R. M. Integral transform solutions of transient natural convection in enclosures with variable fluid properties[J]. International Journal of Heat and Mass Transfer, 2000, 43(21): 3977-3990.

[21] NETO H. L., QUARESMA J. N. N. and COTTA R. M. Natural convection in three-dimensional porous cavities: Integral transform method[J]. International Journal of Heat and Mass Transfer, 2002, 45(14): 3013-3032.
[22] De LiMA G. G. C., SANTOS C. A. C. and HAAG A. $M$. et al. Integral transform solution of internal flow problems based on Navier-Stokes equations and primitive variables formulation[J]. International Journal for Numerical Methods in Engineering, 2007, 69(3): 544-561.

[23] SILVA C. A. M., MACEDO E. N. and QUARESMA J. $\mathrm{N}$. N. et al. Integral transform solution of the NavierStokes equations in full cylindrical regions with streamfunction formulation[J]. International Journal for Numerical Methods in Biomedical Engineering, 2010, 26(11): 1417-1434.

[24] MA J.-K., SU J. and LU C.-H. et al. Integral transform solution of the transverse vibration of an axial moving string $[\mathrm{J}]$. Journal of Vibration, Measurement and Diagnosis, 2006, 26 (117): 104-107.

[25] AN C., SU J. Dynamic response of clamped axially moving beams: Integral transform solution[J]. Applied Mathematics and Computation, 2011, 218(2): 249259.

[26] HUARTE F. J. H. Multi-mode vortex-induced vibrations of a flexible circular cylinder[D]. Doctoral Thesis, London: Imperial College, 2006. 